\title{
The Development of Group Psychology Measurement System based on Computer Multimedia Technology
}

\author{
HuaJie Sui ${ }^{1}$, YaoHui Ye $^{2}$, ZhiBing Zhong ${ }^{3, *}$ \\ ${ }^{1}$ School of Humanity, Jiangxi University of Traditional Chinese Medicine Nanchang, 330004, Jiangxi, \\ China
}

${ }^{2}$ Higher Education Research Office, Jiangxi University of Traditional Chinese Medicine Nanchang, 330004, Jiangxi, China

${ }^{3}$ Psychological Couseling Center, Jiangxi University of Traditional Chinese Medicine Nanchang, 330004, Jiangxi, China

Keywords: Psychological CT, Particle swarm optimization, MATLAB, Curve fitting, Psychological measurement

\begin{abstract}
Mental CT is a kind of new computer psychological measurement technology. In order to realize group measurement function of psychological measurement system, this paper designs particle swarm psychological measurement algorithm, enhancing the system's intelligence community mental health average level calculation and prediction function, realizing performance optimization of psychological measurement system. In order to verify the effectiveness and the reliability of the algorithm, this paper uses MATLAB toolbox to do curve fitting on the psychological test results. From the fitting results, the algorithm can calculate the population average mental health level, which is a reliable method of group psychological measurement.
\end{abstract}

\section{Introduction}

Psychological measurement technology achieves quantity analysis of psychological, and it is not only an important means to evaluate mental psychological health, but also provides a theoretical basis for the psychological activities measurement using computer technology [1,2]. In the computer simulation algorithm design case, there are many skills from biological phenomena, such as artificial neural network is a simplified model of human brain, the genetic algorithm is a simulation of the evolution of genes [3,4]. And the particle swarm optimization is a new design of bionic algorithm, which is a parody of biological system and social system, but also can be called swarm intelligence [5]. Based on local information these simulation systems may result in unpredictable group behavior. When they are applied in psychological measurement process, they can realize the psychological measurement group average calculation.

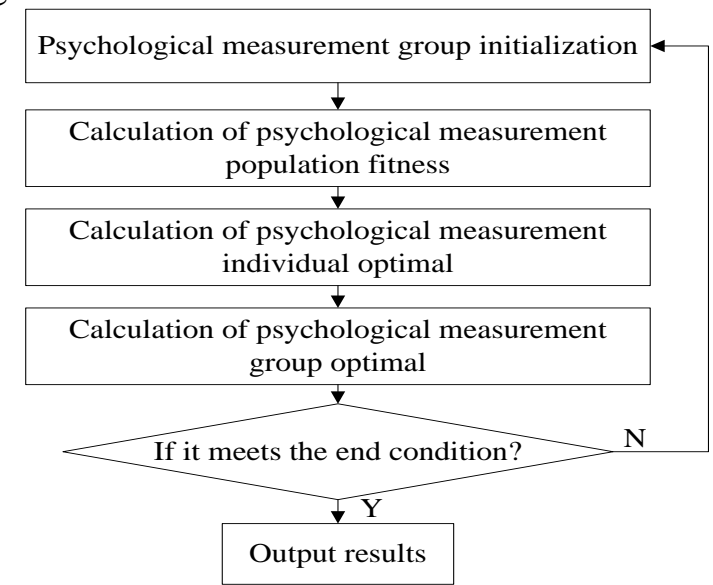

Fig. 1 Design framework of computer group psychological measurement

Figure 1 shows the overall design framework of computer group psychological measurement. The basic step is to first define the psychological measurement group, and initial the velocity and position of group, then calculate the fitness value of psychological measurement group [6,7]. When the fitness 
values satisfy the calculation conditions, first calculate the individual optimal, then calculate the global optimum, if it doesn't satisfy condition, then re- initial psychological measurement group. The output result is done curve fitting by MATLAB, finally the group average mental health is obtained.

\section{Algorithm Design of Group Psychological Measurement Particle Swarm}

Suppose in $D$ dimensional psychological measurement data search space, $N$ psychological measurement results form a community, and the $i$ result represents a $D$ dimension vector.

$X_{i}=\left(x_{i 1}, x_{i 2}, \cdots, x_{i D}\right), i=1,2, \cdots, N$.

Where $i$ psychological measurement speed is also a $D$ dimension vector, denoted as:

$V_{\mathrm{i}}=\left(v_{i 1}, v_{i 2}, \cdots, v_{i D}\right), i=1,2, \cdots 3$.

Where $i$ psychological measurement particle swarm searching optimal position is called individual extreme, denoted as:

$p_{\text {best }}=\left(p_{i 1}, p_{i 2}, \cdots, p_{i D}\right), i=1,2, \cdots, N$.

The best position of psychological measurement particle swarm searching is global optima, denoted as:

$g_{\text {best }}=\left(p_{g 1}, p_{g 2}, \cdots, p_{g D}\right)$.

When finding the two optimal values, particle updates the velocity and position according to the following formula:

$v_{i d}=w * v_{i d}+c_{1} r_{1}\left(p_{i d}-x_{i d}\right)+c_{2} r_{2}\left(p_{g d}-x_{i d}\right), x_{i d}=x_{i d}+v_{i d}$.

Where $c_{1}$ and $c_{2}$ are the study factors, also known as the constant acceleration; $r_{1}$ and $r_{2}$ uniform random number in $[0,1]$. The algorithm process is as follows:

Initialize the particle swarm, including population size $N$, each particle's position $x_{i}$ and velocity $V_{\mathrm{i}}$;

Calculation of each particle's fitness value $F_{i t}[i]$;

For each particle, compare the fitness value $F_{i t}[i]$ with individual extreme $p_{\text {best }}(i)$, if $F_{i t}[i]>p_{\text {best }}(i)$, so replace $p_{\text {best }}(i)$ with $F_{i t}[i]$;

For each particle, compare the fitness value $F_{i t}[i]$ with global extreme $g_{\text {best }}$, if $F_{i t}[i]>p_{\text {best }}(i)$, so replace $g_{\text {best }}$ with $F_{i t}[i]$;

Update the velocity $v_{i}$ and position $x_{i}$ according to the following formula (5);

If it meets the end condition (error is good enough or reaches the maximum number of cycles), it will exit, and otherwise it returns.

In order to achieve the above six steps algorithm, this paper uses the MATLAB to program the calculation $[4,5]$. And the MATLAB programming is as follows:

figure(2)

for $j=1: D$

if $(\operatorname{rem}(D, 2)>0)$

subplot $((\mathrm{D}+1) / 2,2, \mathrm{j})$

else

subplot(D/2,2,j)

end

$\operatorname{plot}\left(x(:, j), b^{* \prime}\right)$; grid on

xlabel('particle')

ylabel(' the initial velocity ')

tInfo=strcat(',char(j+48),' dimension ');

if $(j>9)$

tInfo=strcat(",char(floor(j/10)+48),

char(rem(j,10)+48),' dimension); 
end

End

title(tInfo)

\section{Design of Group Psychological Measurement System based on MATLAB}

In order to verify the validity and reliability of psychological measurement of particle swarm algorithm designed in the second part, this paper uses the commonly used MATLAB software of computer multimedia technology as the core computing software to verify the algorithm $[8,9]$. The MATLAB toolbox opening is as shown in Figure 2.

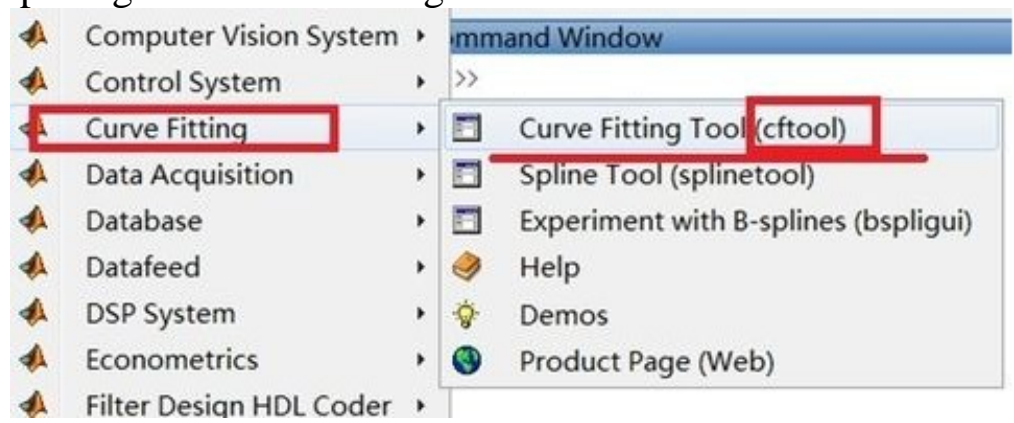

Fig. 2 Opening of the MATLAB toolbox

In the main MATLAB window, click start--toolboxes, it will list all the toolbox MATLAB has been installed, so you can choose the toolbox according to need. Figure 2 shows the curve fitting toolbox of mental health statistical data. Firstly it defines particle initial position of psychological measurement group.

The first dimension

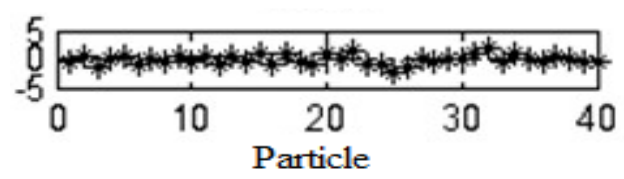

The third dimension

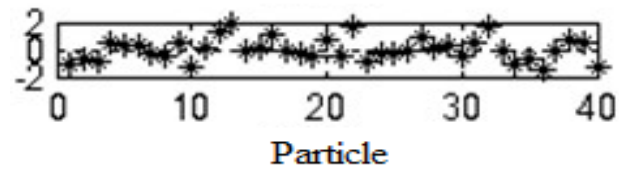

Fig. 3 Group psychological measurement initialization

Figure 3 shows the location initialization of population psychological measurement. The population uses multidimensional initialization mode [10]. Initialize the particle swarm includes population size, each particle's position and velocity. Through calculation the group psychological measurement fitness values are shown in Figure 4.

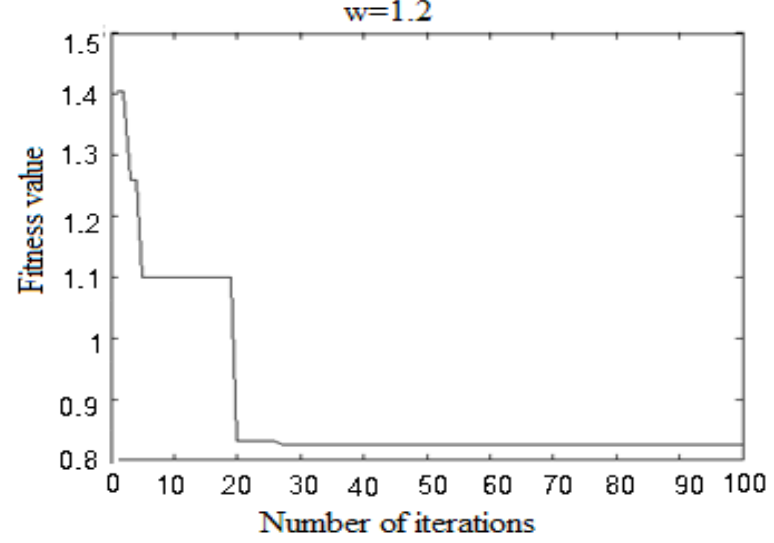

Fig.4 Fitness value computation results 
Figure 4 shows the fitness value of psychological measurement particle swarm by the iteration calculation [11]. When the fitness value is 0.8, the results are optimal. From the chart it can be seen, when iteration step is 20 , it already meets the computational requirements. Through calculation the results are obtained as shown in Table 1.

Table 1. The calculation error statistics

\begin{tabular}{|l|l|l|l|l|}
\hline $\begin{array}{l}\text { Source } \\
\text { variance }\end{array}$ & Square sum & Freedom degree & Mean square & F ratio \\
\hline Factor & 0.43212 & 3 & 0.14404 & 64.4418 \\
\hline Error & 0.04734 & 20 & 0.00236 & \\
\hline Sum & 0.47946 & 23 & & \\
\hline
\end{tabular}

Table 1 shows the results of group effect significant test of psychological measurement. It can be seen from Table $1, F_{0.05}(3,20)=6.10000<64.4418$. In $H_{0}$ parameter changes have significant influence on the results of the program, so the calculating result is valid, final result of calculation uses the MATLAB to do curve fitting, as shown in Figure 5.

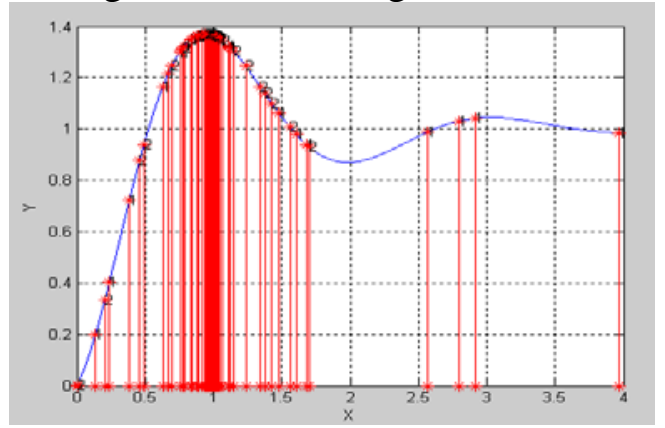

Fig. 5 Group psychological group measuring results

Figure 5 shows the group mental health t results for 800 . The results use curve fitting to map out the general trend of mental health [12]. From the chart it can be seen, the highest health index is 1.4, and the lowest health index is 1.1800 test of the psychological health is at general level.

\section{Summary}

This paper designs particle swarm optimization algorithm of computer psychological measurement, through 6 steps it show the detailed realization of the algorithm, and uses the MATLAB toolbox to program group psychological measurement algorithm. Through the MATLAB simulation, it defines the initial amount of psychological measurement, and the fitness value of group psychological measurement is obtained. Through the curve fitting, the average results of group psychology measurement are obtained. The results show that, the computer group measurement system can calculate average level of group psychological health effectively.

\section{References}

[1] J. Ma. The scientific process of psychological test technology. Journal of Chongqing Academy of Arts and Sciences (SOCIAL SCIENCE EDITION), 2013, 4(6): 112-115.

[2] X.Y. Qiu. The application of multimedia teaching experience in the course of nursing psychology. Higher medical education, 2012, 4(5): 55-58.

[3] X.K. Ma. The application of multimedia technology in nursing psychology teaching effect. Medical equipment information, 2012, 4(6): 102-105.

[4] F.Y. Zhi, J.F. Zhang. A few coping research of national culture from the perspective. Advances in psychological science, 2012, 4(8): 45-48.

[5] J.J. Yan, Y.T. Cui, Y. Qu, X.L. Yang. Research status of psychological elasticity theory. Human resource management, 2012, 5(6): 78-81. 
[6] Miu Shenglong. The domestic research about elasticity orientation. Social psychological science, 2012, 4(5): 56-59.

[7] H.M. Ji. Apriori improved algorithm based on frequent 2 itemset support matrix. Computer engineering, 2013, 4(11): 23-26.

[8] F.X. Sun, S.H. Ni, C. Xie. An improved Apriori algorithm based on matrix Computer simulation, 2013, 4(8): 42-45.

[9] W. Wang, Z.Y. Xin, L. Lei. The relation between values of college students and the coping style, mental health. Journal of Renmin University Education, 2012, 4(4): 66-69.

[10]Y.B. Cheng. Application of the SketchUp software in the conception of architectural design. Harbin Institute of Technology, 2012: 2-16.

[11]Y. Long, X. Li. The building exterior wall thermal insulation energy-saving technology route in Chongqing area. Building energy saving, 2011, 5(6): 21-24.

[12]X.F. Zhang, Y.C. Zhou. BIM technology application field and its prospect analysis based on IFC standard. Building science, 2012, 4(10): 94-97. 